\title{
GUIDELINES
}

\section{Guidelines for the ethical conduct of medical research involving children}

\author{
Royal College of Paediatrics and Child Health: Ethics Advisory Committee
}

These guidelines are written for everyone involved in the planning, review, and conduct of research with children. The Royal College of Paediatrics and Child Health's first guidelines (then the British Paediatric Association) were published in 1980. Since then, there has been significant progress in the understanding of children's interests, in legal requirements, and in the proper regulation of research. The revised guidelines take account of such developments. General guidelines relating to all medical research provide an essential background to this document on research with children. ${ }^{1-9}$ These guidelines are based on six principles:

(1) Research involving children is important for the benefit of all children and should be supported, encouraged and conducted in an ethical manner

(2) Children are not small adults; they have an additional, unique set of interests

(3) Research should only be done on children if comparable research on adults could not answer the same question

(4) A research procedure which is not intended directly to benefit the child subject is not necessarily either unethical or illegal

(5) All proposals involving medical research on children should be submitted to a research ethics committee

(6) Legally valid consent should be obtained from the child, parent or guardian as appropriate. When parental consent is obtained, the agreement of school age children who take part in research should also be requested by researchers.

The special implications of fetal research are considered by the Polkinghorne Report. ${ }^{10}$

Value of ethical research with children Medical research involving children is an important means of promoting child health and wellbeing. Such research includes systematic investigation into normal childhood development and the aetiology of disease, as well as careful scrutiny of the means of promoting health and of diagnosing, assessing, and treating disease. It is also important to validate in children the beneficial results of research conducted in adults.

Research with children is worthwhile if each project:
- has an identifiable prospect of benefit to children

- is well designed and well conducted

- does not simply duplicate earlier work

- is not undertaken primarily for financial or professional advantage

- involves a statistically appropriate number of subjects

- eventually is to be properly reported.

Comprehensive registers such as the $\mathrm{Na}$ tional Perinatal Epidemiology Unit's register of perinatal research and the National Research Register help to promote high standards. They publicise worthwhile projects and good practice; they help to prevent unnecessary duplication; and by recording unpublished work they provide valuable information.

\section{Children's interests}

Children are unique as a research group for many reasons. They are the only people, in British law, on whose behalf other individuals may consent to medical procedures. Many children are vulnerable, easily bewildered and frightened, and unable to express their needs or defend their interests. Potentially with many decades ahead of them, they are likely to experience, in their development and education, the most lasting benefits or harms from research.

To facilitate both the child's health care and longer term research, general practitioners should be notified of all research on their paediatric patients. Long term follow up of research interventions may be of particular benefit to child subjects. Yet this means still more intrusion into their lives, as records are shared and computerised. Children may be less able than adults to challenge records about themselves. There is therefore a duty on researchers to respect confidentiality, and keep up to date with data protection and legislation on access to health records.

More needs to be known about how children are affected by their experiences as patients and research subjects, and what support they need. It is desirable to encourage psychosocial research conducted independently and in conjunction with physiological research. Research will then further the task of caring for the whole child within the family.

Research shares this task when it is more than research on children, and is research with 
them, learning from their responses and attending to their interests as perceived by the child and parents. ("Parents" in these guidelines refers to parents, guardians, or adults legally entitled to give consent on the child's behalf.) This partnership should accord with the Declaration of Helsinki in that concern for the interests of the subject must always prevail over those of science and society.

Must the research involve children?

In principle, the informed and willing consent of human research subjects should be sought whenever possible. Yet there are complications in obtaining the consent of minors. Research and innovative treatment on humans should only be undertaken after adequate basic research. Research with children should be undertaken only if work with adults is clearly not feasible. When a choice of age groups is possible, older children should be involved in preference to younger ones, although much valuable research can only be done with younger children and babies.

Some treatments, such as organ transplantation, may involve a range of procedures. Each separate new procedure should be tested with informed willing adults when possible, with time to assess at least the medium term effects, before the procedure is attempted with children. The urgent desire to offer babies and children the potential benefits of medical innovation is laudable. Yet childhood is a vulnerable, formative time, when harms can have serious impact as well as being potentially long lasting. Potential harms should therefore be assessed carefully before children are put at risk.

Increasingly, research experience is regarded as an essential qualification for promotion in medicine. Research work can offer valuable training that may improve the quality of doctors' clinical practice. An inquiring mind disciplined to test hypotheses by the approved canons of research while sensitive to the vulnerability of child patients should be seen as a valued professional asset in a paediatrician.

However, great care should be exercised by supervisory senior staff over the choice of research projects. The criteria for worthwhile research, listed above, must govern the selection of projects whether primarily undertaken as part of medical training or for the advancement of knowledge.

\section{Potential benefits, harm, and cost}

There are no general statutory provisions covering research on human beings. In the absence of relevant case law, earlier cautions against research on minors that offer no direct benefit to the child subject ${ }^{11}$ have been replaced by qualified support. ${ }^{5}{ }^{12}$ This has not been challenged in the courts. The attempt to protect children absolutely from the potential harms of research denies any of them the potential benefits. We therefore support the premise that research that is of no intended benefit to the child subject is not necessarily unethical or illegal. Such research includes observing and measuring normal development, assessing diagnostic methods, the use of "healthy volunteers" and of placebos in controlled trials.

The importance of evaluating potential benefits, harms, and costs in research on human beings, and ways of doing so, ${ }^{7}$ have been discussed repeatedly. A summary of discussion points is included in these guidelines to illustrate how complex such evaluations can be. Our aim, rather than to provide answers, is to list questions for researchers and ethics committees to consider.

\section{Assessment of potential benefit includes reviewing estimates of:}

Magnitude

- How is the knowledge gained likely to be used?

- In research into treatment how severe is the problem which the research aims to alleviate?

- How common is the problem?

Probability

- How likely is the research to achieve its aims?

Beneficiaries

- Is the research intended to benefit the child subjects, and/or other children?

Resources

- Will potential benefits be limited because they are very expensive, or require unusually highly trained professionals?

\section{Assessment of potential harm included} estimates of:

Types of intervention

- How invasive or intrusive is the research? (psychosocial research should be assessed as carefully as physical research)

Magnitude

- How severe may the harms associated with research procedures be?

Probability

- How likely are the harms to occur?

Timing

- Might adverse effects be brief or long lasting, immediate or not evident until years later?

Equity

- Are a few children drawn into too many projects simply because they are available?

- Are researchers relying unduly on children who already have many problems?

Interim finding

- If evidence of harm in giving or withholding certain treatment emerges during the trial, how will possible conflict between the interests of the child subjects and of valid research be managed?

\section{Assessment of potential harm also} includes reviewing personal estimates Children's responses are varied, often unpredictable, and alter as children develop, so that generalisations about risk tend to be controversial. A procedure that does not bother one child arouses severe distress in another. Researchers sometimes underestimate high risk of pain if the effects are brief, whereas the child or parents may consider the severe transient pain 
is not justified by the hoped for benefit. There is evidence that tolerance of pain increases with age and maturity when the child no longer perceives medical interventions as punitive. ${ }^{13-15}$

Some potential harms may not be obvious without careful consideration of their consequences. For example, with research into serious genetic disorders that present in adult life, presymptomatic diagnosis in a child, while it may be beneficial, may also have very harmful effects, and may affect the child's opportunities and freedom of choice. ${ }^{16}$

\section{Risks may be estimated as minimal, low or high}

Minimal (the least possible) risk describes procedures such as questioning, observing, and measuring children, provided that procedures are carried out in a sensitive way, and that consent has been given. Procedures with minimal risk include collecting a single urine sample (but not by aspiration), or using blood from a sample that has been taken as part of treatment.

Low risk describes procedures that cause brief pain or tenderness, and small bruises or scars. Many children fear needles and for them low rather than minimal risks are often incurred by injections and venepuncture.

High risk procedures such as lung or liver biopsy, arterial puncture, and cardiac catheterisation are not justified for research purposes alone. They should be carried out only when research is combined with diagnosis or treatment intended to benefit the child concerned.

We believe that research in which children are submitted to more than minimal risk with only slight, uncertain or no benefit to themselves deserves serious ethical consideration. The most common example of such research involves blood sampling. Where children are unable to give consent, by reason of insufficient maturity or understanding, their parents or guardians may consent to the taking of blood for non-therapeutic purposes, provided that they have been given and understand a full explanation of the reasons for blood sampling and have balanced its risk to their child. Many children fear needles, but with careful explanation of the reason for venepuncture and an understanding of the effectiveness of local anaesthetic cream, they often show altruism and allow a blood sample to be taken. We believe that this has to be the child's decision. We believe that it is completely inappropriate to insist on the taking of blood for nontherapeutic reasons if a child indicates either significant unwillingness before the start or significant stress during the procedure.

Despite careful selection, children in clinical trials have social and emotional problems that are mainly unpredictable. ${ }^{17}$ Provision for necessary, continuing, emotional support should be built into the research design.
Assessment of potential cost includes reviewing:

Resources

- How much medical, nursing and other professional time is required for informing and supporting families, and for collecting data?

- Are sufficient staff available without prejudicing the care of patients?

- Are the costs of reducing and preventing harms included in the protocol (such as information material for staff and families, local anaesthetic cream (for example, EMLA), autolet)

- How much family time is required for collecting data, or attending clinics?

- How will their extra expenses be paid?

- Are reasonable costs allowed for collecting, collating, and analysing data, for writing and disseminating the reports, and for informing research subjects of the results?

Statistics

- Are enough children involved to make a statistically valid sample, and to allow for withdrawals during longer studies?

- Is the planned number of subjects unnecessarily high?

Inconvenience

- How much inconvenience to families is justified (such as extra visits to clinics)?

- All medical research, whether or not associated with therapy, requires careful evaluation, as well as the safeguards described in the next two sections.

\section{Research ethics committees}

As assessment of benefit and harm is complex, children are best protected if projects are reviewed at many levels, by researchers, funding and scientific bodies, research ethics committees, the research assistants and nurses working with child subjects, the children, and their parents. Everyone concerned (except young children) has some responsibility.

Ethics is about good practice, and each research ethics committee considering a project involving children should be advised by people with a close, practical knowledge of babies and children, such as a registered sick children's nurse. They may be research ethics committee members, persons co-opted, or members of a subcommittee.

Given that valuable research can range from the descriptive using qualitative methods to that requiring statistical analysis, research ethics committees need to have or to co-opt members with a breadth of experience adequate to assess such research.

Multicentre research ethics committees (MRECs) have the task of reviewing all research taking place in five or more centres, and protocols approved by the MREC cannot be amended by a local research ethics committee (LREC). It is therefore particularly important that multicentre studies in children are always assessed after such advice and that LRECs are advised accordingly. LRECs considering multicentre protocols should ensure that there are no local objections to the study 
(for example, over researched groups, ethnic factors, research facilities, local investigators).

The duties of LRECs have been clearly described. ${ }^{34}$ Those of MRECs have also been outlined..$^{18}$ It is important that committees are satisfied that each project:

- sets out to answer a useful question or questions

- is designed in the best possible way to answer the questions

- will work in practice (such as in the safety of drugs and techniques, age appropriate interventions, and prevention of too many studies being carried out in one ward).

Both MRECs and LRECs may also wish to know how researchers plan to monitor and respond to any signs of distress in child subjects. This may involve helping children to withdraw from the study. LRECs have the additional responsibility of monitoring the progress of studies. ${ }^{19}$

Research ethics committees are faced with the paradox of trying to be both stringent assessors and an approachable forum for helping researchers to resolve problems. They have to compromise between aiming for the perfect protocol in advance and encouraging researchers to respond to families' unpredictable responses, which may require changes in the research design later on.

\section{Consent and assent}

"Consent", in this section, describes the positive agreement of a person; "assent" refers to acquiescence. The law relating to research on children (children defined by law as those under 18 years) has never been clearly established. The application of general principles indicates that, where children have "sufficient understanding and intelligence to understand what is proposed", it is they and not their parents whose consent is required by law. ${ }^{20} \mathrm{~A}$ reasoned refusal by a child to participate in research is likely to be taken as evidence of such understanding, and it would be unwise to rely on parental consent in such circumstances. If the child is insufficiently mature to consent, then valid parental consent must be obtained.

The physical integrity of children, as of all other people, is protected by law. Unless they, or their parents or guardians acting on their behalf, agree to it, nothing can be done lawfully that involves touching them. Research with children must normally be carried out only with the consent of parent, guardian or child. Some research based on observation, collating information from notes and tests already performed for therapeutic purposes may, however, be permissible without consent because it does not involve touching the child.

A general exception to the requirement for consent is the provision of medical care in an emergency. If emergency medical, surgical, and neonatal care are to be improved, research is necessary. On many, but not all, such occasions, it may be impracticable, or meaningless, to attempt immediately to obtain informed consent for the proposed research procedures from parents or guardians. To require such an attempt always to be made could also inhibit much potentially valuable research.

Provided, therefore, that the specific approval of a research ethics committee has been obtained for the project overall, it would be ethical to carry out research on children on such occasions of extreme urgency without obtaining consent. It is possible, however, that it would still be unlawful if the research were not expected to benefit the child in question, although legal action would be unlikely. The parents or guardians and, where appropriate, the child must be informed about the research as soon as possible afterwards: a requirement in ethics as in courtesy.

Parental consent will probably not be valid if it is given against the child's interests. This means that parents can consent to research procedures that are intended directly to benefit the child, but that research that does not come into this category can only be validly consented to if the risks are sufficiently small to mean that the research can be reasonably said not to go against the child's interests. ${ }^{21}$ Even when it is not legally required, researchers should obtain the assent or agreement of school age children to their involvement in the research, and should always ensure that the child does not object.

Legally valid consent is both freely given and informed. For consent to be freely given researchers must:

- offer families no financial inducement, although expenses should be paid

- exert no pressure on families

- give them as much time as possible (some days for a major study) to consider whether to take part in the project

- encourage families to discuss the project with-for example, their relatives, or primary health carers

- tell them that they may refuse to take part, or may withdraw at any time, even if they have signed a consent form

- say that they need not give a reason for withdrawing, although their reason may help the researchers and other children in the study

- assure them that the child patient's treatment will not be prejudiced by withdrawal from the research

- encourage parents to stay with their child during procedures

- respond to families' questions, anxiety or distress throughout the study.

Consent is not a single response; it involves willing commitment that may falter during a long, difficult project. Families may need to be supported and informed frequently. Children's ability to consent develops as they learn to make increasingly complex and serious decisions. Ability may relate to experience rather than to age, and even very young children appear to understand complex issues. They should therefore be informed as fully as possible about the research in terms they can understand.

For consent to be informed, researchers must discuss with families:

- the purpose of the research 
- whether the child stands to benefit directly and, if so, how; the difference between research and treatment

- the meaning of relevant research terms (such as placebos)

- the nature of each procedure, how often or for how long each may occur

- the potential benefits and harms (both immediate and long term)

- the name of a researcher whom they can contact with inquiries

- the name of the doctor directly responsible for the child

- how children can withdraw from the project. Researchers will also:

- willingly explain and answer questions throughout the project

- ensure that other staff caring for child subjects know about the research, and can also explain it if necessary

- give clearly written leaflets for families to keep

- should report the results of research to the families involved.

When explaining relevant terms, researchers need to discuss with families the consent implications. For example, consenting to a double blind randomised trial means not minding which of a choice of treatments the child will have, and that neither the family nor their doctor will know which treatment has been given until the trial has been completed.

These guidelines are designed to benefit children who take part in research, children who may be helped by the research findings, and medical research itself. Researchers who observe high standards will continue to enjoy public support and cooperation.

These guidelines were produced initially by the Ethics Advisory Committee of the British Paediatric Association in $1992^{\star}$ and have been modified and updated by the Royal College of Paedihave been modified and updated by the Royal College of Paedi atrics and Child Health Ethics Advisory Committee in $1999^{+}$. ${ }^{\star}$ Chairman, Professor C Normand; Members, Dr P Alder-
son, Miss G Brykczynska, Professor R Cooke, Professor The son, Miss G Brykczynska, Professor R Cooke, Professor The
Rev G Dunstan CBE, Professor Dame J Lloyd DBE, Mr J Rev G Dunstan CBE, Professor Dame J Lloyd DBE
Montgomery, Dr R Nicholson, Professor M Pembrey.

Montgomery, Dr R Nicholson, Professor M Pembrey.
${ }^{+}$Chairman, Professor N McIntosh; Members, Mr P Bates,

${ }^{+}$Chairman, Professor N McIntosh; Members, Mr P Bates,
Miss G Brykczynska, Professor The Rev G Dunstan CBE, Dr A Goldman, Professor D Harvey, Dr V Larcher, Dr D McCrae, D A McKinnon, Dr M Patton, Dr J Saunders, Mrs P Shelley.

Royal College of Physicians. Research on healthy volunteers. London: The College, 1988.

2 Royal College of Physicians. Research involving patients. London: The College, 1990.

3 Royal College of Physicians. Guidelines on the practice of ethics committees involved in medical research involving human subjects. 3rd ed. London: The College, 1996.

4 NHS Management Executive. Guidelines on local research ethics committees. London: NHS Management Executive, 1991 .

5 World Medical Association. The declaration of Helsinki. Ferney-Voltaire, France: The Association, 1996; revised in Ferney-Voltaire, France:

6 United Kingdom Central Council on Nursing, Midwifery and Health Visiting. Exercising accountability: A framework to assist nurses, midwives, and health visitors to consider ethical aspects of professional practice. London: The Council, 1989.

7 Nicholson R, ed. Medical research with children: ethics, law and practice. Oxford: Oxford University Press, 1986.

8 National Commission for the Protection of Human Subjects of Biomedical and Behavioral Research. Research involving
children: report and recommendations. Washington DC: Department of Health, Education and Welfare, 1977.

9 Alderson P. Choosing for children: parents' consent to surgery. Oxford: Oxford University Press, 1990.

10 Review of the guidance on the research use of fetuses and fetal material (The Polkinghorne Report). London: Her Majesty's Stationery Office, $1989 \mathrm{Cm} 762$.

11 Medical Research Council. Responsibility in investigations on human subjects: In: Report of the Medical Research Coun-
cil for the year 1962-63: 21-5. London: Her Majesty's cil for the year $1962-63$ :
Stationery Office, 1964.
12 Dworkin G. Legality of consent to non-therapeutic medical research on infants and young children. Arch Dis Child 1978;51:443-6.

13 Haslam D. Age of perception of pain. Psychological Science 1969;15:86-7

14 Petrillo D, Danger P. Emotional care of hospitalised children New York: Lippincott, 1980

15 Jacox A, ed. Pain: a sourcebook for nurses and other health professionals. Boston: Little Brown, 1977.

16 British Paediatric Association Ethics Advisory Committee. Testing children for late onset genetic disorders. London: BPA Statement, 1996.

17 Kinmonth A, Lindsay M, Baum J. Social and emotional complications in a clinical trial among adolescents with diabetes mellitus. BMF 1983;286:952-4

18 NHS Executive. Ethics committee review of multi-centre research. HSG(97)23. London: Department of Health, 1997.

19 International Conference on Harmonisation of Technical Requirements for Registration of Pharmaceuticals for Human Use (ICH Harmonised Tripartite Guidelines). Guideline for good clinical practice. Geneva: IFPMA, 1997.

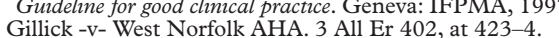

21 S -v- S. 3 All ER 107, at 111.

\section{Commentary}

The Ethics Committee of the British Paediatric Association (BPA), now the Royal College of Paediatrics and Child Health (RCPCH), has prepared guidelines on the planning, conduct, and review of research on children. Two of their basic principles are that "research involving children is important for the benefit of all children" and that "a research procedure not intended directly to benefit the child subject is not necessarily either unethical or illegal". Research is necessary to ensure that children receive fully informed care.

Analysis of blood is an essential part of many research programmes-for example, the determination of nutritional status and the evaluation of therapeutic drugs. Taking blood is often painful, it sometimes leaves a worrying bruise, and the experience can be distressing. Debate both in and out of the Councils of the $\mathrm{BPA} / \mathrm{RCPCH}$ has centred on whether taking blood from children poses a minimal or a low risk. Procedures that have a low risk are usually inappropriate if the child involved is unlikely to benefit from the experience.

Blood taken by a skilled person poses only a minimal risk of physical harm, except possibly the taking of a separate sample of blood from a very immature infant. It is the pain and distress and the memory of it that might cause more than minimal harm.

Taking blood from children can be a positive experience. Children, like adults, are capable of being generous and doing something worthwhile even if it means they experience discomfort. Parents when they are fully informed usually consent to their babies experiencing the brief pain of blood taking if the investigation might be to the benefit of other infants. It seems to me that for blood taking to be a minimal risk, it is important that all concerned know what is happening, that the moment when the blood is taken is appropriate, that the procedure is skilfully performed, and that all steps are taken to reduce the amount of pain the child experiences-for example, by using local anaesthetic creams.

Some children, however, like some adults are frighten of needles and the sight of blood. The guidance originally stated that "Many children fear needles and to them low rather than 
minimal risks are often incurred by injection and venepuncture". It now says "Many children fear needles, but with careful explanation of the reason for the venepuncture and an understanding of the effectiveness of anaesthetic cream, they often show altruism, and allow a blood sample to be taken". I welcome the move to a more positive position. It is in the common interest of children. But it makes it more, rather than less, necessary for research workers to be able to recognise when a child is very upset whether by the thought of the procedure or at the time of the procedure and to accept this distress as genuine dissent from being involved. The child's feelings are not to be sacrificed.

I hope that the number of children who get very upset at the thought of needles or the sight of blood will fall as accident and emergency departments strive to ensure that when children attend for treatment, the experience enhances rather than undermines their confidence in modern clinical care.

PROFESSOR SIR DAVID HULL Emeritus Professor 